Georgian Mathematical Journal

Volume 13 (2006), Number 1, 363-382

\title{
SELFADJOINT OPERATORS AND GENERALIZED CENTRAL ALGORITHMS IN FRECHET SPACES
}

\author{
SOSO TSOTNIASHVILI AND DAVID ZARNADZE
}

\begin{abstract}
The paper gives an extension of the fundamental principles of selfadjoint operators in Fréchet-Hilbert spaces, countable-Hilbert and nuclear Fréchet spaces. Generalizations of the well known theorems of von Neumann, Hellinger-Toeplitz, Friedrichs and Ritz are obtained. Definitions of generalized central and generalized spline algorithms are given. The restriction $A^{\infty}$ of a selfadjoint operator $A$ defined on a dense set $D(A)$ of the Hilbert space $H$ to the Frechet space $D\left(A^{\infty}\right)$ is substantiated. The extended Ritz method is used for obtaining an approximate solution of the equation $A^{\infty} u=f$ in the Frechet space $D\left(A^{\infty}\right)$. It is proved that approximate solutions of this equation constructed by the extended Ritz method do not depend on the number of norms that generate the topology of the space $D\left(A^{\infty}\right)$. Hence this approximate method is both a generalized central and generalized spline algorithm.

Examples of selfadjoint and positive definite elliptic differential operators satisfying the above conditions are given. The validity of theoretical results in the case of a harmonic oscillator operator is confirmed by numerical calculations.
\end{abstract}

2000 Mathematics Subject Classification: 47B25, 65D15, 41A65, 65J10, 65L60, 68Q25.

Key words and phrases: Selfadjoint operator, best approximation, generalized central algorithm, extended Ritz method, energetic Fréchet space.

\section{INTRODUCTION}

Symmetrical and selfadjoint operators in Fréchet-Hilbert, countable-Hilbert and nuclear Fréchet spaces were introduced and investigated in [1-5]. Since the class of these spaces contains products of Hilbert space sequences, the theory considered in this paper actually includes the theory of selfadjoint operators in spaces of functions with a countable number of arguments, but differs from the theory constructed in [6]. On the basis of the results announced in [3] and proved below, in [4] the Ritz method was extended for equations with positive definite operators in the above-indicated Fréchet spaces.

In this paper the Ritz method is used to obtain an approximate solution of the equation

$$
A u=f
$$

with a positive definite operator $A$ in the Hilbert space $H$, assuming that the right-hand part is sufficiently smooth. We first consider the restriction of equation (1) in the countable-Hilbert space $D\left(A^{\infty}\right)[7-8]$ (the space $D\left(A^{\infty}\right)$ coincides 
with the space $C^{\infty}(A)\left([9]\right.$, p. 345) which is the set of $C^{\infty}$-elements of the operator $A$ ). Then we prove that the restriction of the operator $A$ denoted by $A^{\infty}$ is continuous and selfadjoint in the space $D\left(A^{\infty}\right)$. Due to this notation, spaces $D\left(A^{\infty}\right)$ acquire a new meaning that differs from the classical case where $D\left(A^{\infty}\right)$ was the whole symbol, where $A^{\infty}$, if taken separately, meant nothing. From now on the symbol $D\left(A^{\infty}\right)$ will also denote the definition domain of the operator $A^{\infty}$ which obviously coincides with the space $D\left(A^{\infty}\right)$. Since the space $D\left(A^{\infty}\right)$ is everywhere dense in its strongly adjoint space $D\left(A^{\infty}\right)_{\beta}^{\prime}, A^{\infty}$ admits a continuous extension to it. Further, we prove that for the equation

$$
A^{\infty} u=f
$$

the extended Ritz method converges in the energetic space $E_{A^{\infty}}$ of the operator $A^{\infty}$. In that case, the space $E_{A^{\infty}}$ coincides with the space $D\left(A^{\infty}\right)$ and its topology is stronger than that of the energetic space $H_{A}$ of the operator $A$. The obtained results are applied to an equation with strongly degenerating elliptical operators and with a harmonic oscillator operator in the Hilbert space $L^{2}(R)$. For the latter operator, the space $D\left(A^{\infty}\right)$ coincides with the Schwartz space $S(R)$ of fast decreasing functions [7]. Therefore if we take as base functions the eigenfunctions $\left\{\varphi_{j}\right\}$ of this operator, i.e., the Hermite functions, then the sequence of approximate solutions converges to an exact solution in the space $S(R)$, the topology of which is stronger than that of the Sobolev space. Our numerical experiments confirm the validity of the theoretical results.

We used the obtained results also for the operator $K^{-1}$ in the ill-posed problem

$$
K u=f
$$

in the Hilbert space $H$ with a compact selfadjoint operator $K: H \rightarrow H$. This enabled us to immediately establish that the restriction of the operator $K^{-1}$ is continuous and selfadjoint in the Fréchet space $D\left(K^{-\infty}\right)=D\left(\left(K^{-1}\right)^{\infty}\right)$. Since the space $D\left(K^{-\infty}\right)$ is everywhere dense in its strongly adjoint space $D\left(K^{-\infty}\right)_{\beta}^{\prime}$, it admits a continuous extension to it. This means that in the latter space initial equation (3) is strongly well-posed. The weak well-posedness of these problems was proved in $[10-11]$.

Section 1 contains the definitions of selfadjoint and positive definite operators in Fréchet spaces, the topologies of which are generated by sequences of Hilbertian seminorms. For such spaces we generalize the well known theorems of Neumann, Hellinger-Toeplitz and Friedrichs. We also give the definition of an energetic space of a positive definite operator and represent this space as the projective limit of a sequence of energetic spaces of projection operators in Hilbert spaces.

In Section 2, we justify our consideration of the restriction of the selfadjoint operator $A$ defined on a dense subset $D(A)$ of the Hilbert space $H$ to the countable-Hilbert space $D\left(A^{\infty}\right)$. Using the general results obtained in Section 1 we prove that the above-mentioned restriction $A^{\infty}$ to the Fréchet space $D\left(A^{\infty}\right)$ is continuous and selfadjoint. Moreover, we prove that if the operator $A$ is positive definite in $H$ and has a purely pointwise spectrum, then $A^{\infty}$ is an 
isomorphism of the space $D\left(A^{\infty}\right)$ onto and its energetic space $E_{A^{\infty}}$ coincides with $D\left(A^{\infty}\right)$.

In Section 3, the extended Ritz method is used to obtain an approximate solution of equation (2) in the Fréchet space $D\left(A^{\infty}\right)$, where $A^{\infty}$ is again the restriction of the operator $A$ with a purely pointwise spectrum. We choose eigenfunctions of the operator $A$ as base functions and prove that the subspaces spanned over the first eigenvector $m$ possess an orthogonal complement in the Fréchet space $D\left(A^{\infty}\right)$. This means that approximate solutions do not depend on the number of norms that generate the topology of the space $D\left(A^{\infty}\right)$. It is shown that a sequence of approximate solutions is convergent in the space $D\left(A^{\infty}\right)$, the topology of which is stronger than that of the initial Hilbert space (Theorem 4). We give the definitions of a generalized central and generalized spline algorithm and prove that the algorithm given in Theorem 4 is both generalized central and generalized spline. An analogous result was obtained in [12] (see also [13]). Examples of selfadjoint and positive definiite elliptical differential operators satisfying the conditions of Theorem 4 are given. Further, it is indicated that the obtained results can be applied to the well known Lagrange, Legendre and Tricomi differential operators

The results of numerical experiments are presented, which confirm the validity of theoretical investigations in the case of a harmonic oscillator operator.

At the end of Section 3 the obtained results are applied to the ill-posed problem (3) in a Hilbert space. In particular, by applying them to the inverse compact selfadjoint operator we prove the strong and the weak well-posedness of equations with such operators. These results are also applied to operators mapping a separable Hilbert space into the same space and admitting a singular decomposition.

\section{Extension of the von Neumann, Hellinger-Toeplitz and Friedrichs Theorems to the CASE of Symmetrical Operators IN FRÉCHET SPACES}

Let us recall some definitions from [3]. Let $E$ be the complex Fréchet space with an increasing sequence of Hilbertian seminorms $\left\{\|\cdot\|_{n}\right\}$, where $\|x\|_{n}=$ $(x, x)_{n}^{1 / 2}$ for each $x \in E$ and $(\cdot, \cdot)_{n}$ is a semiscalar product on $E$. The term "semiscalar product" means that the inner product $(\cdot, \cdot)_{n}$ has no property $(x, x)_{n}=0 \Rightarrow x=0$. Let $A$ be a linear operator with the dense definition domain $D(A)$ which, for the sake of simplicity, in what follows will be called a dense linear operator. If for $y \in E$ there exists an element $y^{*}$ such that

$$
(A x, y)_{n}=\left(x, y^{*}\right)_{n}
$$

for each $x \in D(A)$ and $n \in N$, then using the equality $A^{*} y=y^{*}$ we define the operator $A^{*}: H \rightarrow H$ which we call the Hilbert adjoint operator to $A$. In other words, $D\left(A^{*}\right)$ consists of $y \in E$ for which there exists a vector $y^{*}$ such that (4) is fulfilled for all $x \in D(A)$ and $n \in N$. It should also be noted that the operator $A^{*}$ differs from the ordinary topological adjoint operator $A^{\prime}$ [3]. 
The dense operator $A$ is called symmetrical if $A \subset A^{*}$, i.e., if the adjoint operator $A^{*}$ is an extension of $A$.

The symmetrical operator $A$ can also be defined in the following equivalent manner: the operator $A$ is called symmetrical if

$$
(A x, y)_{n}=(x, A y)_{n}
$$

for all $x, y \in D(A)$ and $n \in N$.

The symmetrical operator $A$ is called selfadjoint if $A=A^{*}$.

The symmetrical operator $A$ is called positive definite if for each $n \in N$ there exists $\gamma_{n}>0$ such that

$$
(A x, x)_{n} \geq \gamma_{n}^{2}(x, x)_{n} \quad \text { for all } \quad x \in D(A) \quad \text { and } \quad n \in N .
$$

Let us now give the generalizations of the von Neumann theorems on symmetrical and selfadjoint operators.

Theorem 1. Let $E$ be the Fréchet space with a generating increasing sequence of Hilbertian seminorms $\left\{\|\cdot\|_{n}\right\}$. Then the following statements are true:

a) The symmetrical operator $A$ defined on the whole space $D(A)=E$ is selfadjoint and continuous. Moreover, if $E$ is a Fréchet-Hilbert space, then $A \in L_{0}(E)$, i.e., for each $n \in N$ there exists $C_{n}>0$ such that

$$
\|A x\|_{n} \leq C_{n}\|x\|_{n} \quad \text { for all } x \in E .
$$

b) The symmetrical operator $A$ with the dense image $R(A)$ possesses the symmetrical inverse operator $A^{-1}$. Moreover, if $A$ is positive definite, then $A^{-1} \in L_{0}(E)$.

c) Let $A$ be the symmetrical operator with the dense image $R(A)$ in $E$. Then $A$ is selfadjoint if and only if $A^{-1}$ is selfadjoint.

d) The symmetrical operator $A$, the image $R(A)$ of which coincides with $E$, is selfadjoint.

Proof. a) By the symmetry of $A$ we have $D(A) \subset D\left(A^{*}\right)$, but $D(A)=E$ and therefore $D\left(A^{*}\right)=E$, i.e., $A=A^{*}$. Let us prove that the operator $A$ is closed. Indeed, let $u_{m} \rightarrow u, A u_{m} \rightarrow v_{0}$ for $m \rightarrow \infty$. Then for each $n \in N$ and $v \in E$ we have

$$
\left(v_{0}, v\right)_{n}=\lim _{m \rightarrow \infty}\left(A u_{m}, v\right)_{n}=\lim _{m \rightarrow \infty}\left(u_{m}, A v\right)_{n}=(u, A v)_{n}=(A u, v)_{n}
$$

i.e., $v_{0}=A u$. Thus the operator $A$ is closed and therefore continuous.

If $E$ is a Fréchet-Hilbert space, then the factor space $E / \operatorname{Ker}\|\cdot\|_{n}$ is a Hilbert space with the scalar product $\left\langle K_{n} x, K_{n} y\right\rangle_{n}=(x, y)_{n}$ and associated norm $\left\|\widehat{K_{n} x}\right\|_{n}=\left\langle K_{n} x, K_{n} x\right\rangle^{1 / 2}=\|x\|_{n}$ for each $x, y \in E$, where $K_{n}: E \rightarrow E / \operatorname{Ker}\|\cdot\|_{n}$ is the canonical mapping. Then the operator $A_{n}$ defined by the equality

$$
A_{n}\left(K_{n} x\right)=K_{n}(A x)
$$

is positive definite on the whole Hilbert space $E_{n}=\left(E / \operatorname{Ker}\|\cdot\|_{n}, \widehat{\|\cdot\|_{n}}\right)$. 
The condition for the operator $A_{n}$ to be correct is as follows: for any $x_{1}$ and $x_{2}$ from $E$ the condition $x_{1}-x_{2} \in \operatorname{Ker} K_{n}$ implies $A\left(x_{1}-x_{2}\right) \in \operatorname{Ker} K_{n}$. The operator $A_{n}$ is symmetrical. Indeed, let $K_{n} x, K_{n} y \in E_{n}$. Then

$$
\begin{gathered}
\left\langle A_{n}\left(K_{n} x\right), K_{n} y\right\rangle=\left\langle K_{n}(A x), K_{n} y\right\rangle=(A x, y)_{n}=(x, A y)_{n} \\
=\left\langle K_{n} x, K_{n}(A y)\right\rangle=\left\langle K_{n} x, A_{n}\left(K_{n} y\right)\right\rangle .
\end{gathered}
$$

Therefore, by virtue of the classical Hellinger-Toeplitz theorem, $A_{n}$ is continuous for each $n \in N$, i.e., $A \in L_{0}(E)$.

In the sequel we will call the operator $A_{n}$ the projection of the operator $A$ onto the factor space $\left(E / \operatorname{Ker}\|\cdot\|_{n}, \widehat{\|\cdot\|_{n}}\right)$, keeping in mind that this definition is meaningful for the operator $A$ acting from the Fréchet space $E$ into.

b) Let us prove that the equality $A x=0$ implies $x=0$. Indeed, for all $y \in D(A)$ and $n \in N$ we have $(x, A y)_{n}=(A x, y)_{n}=0$. Hence, by the density of $R(A)$ in $E$, we obtain that $x \in \operatorname{Ker}\|\cdot\|_{n}$ for each $n \in N$, i.e., $x=0$. Therefore there exists the inverse operator $A^{-1}$ with the dense domain $D\left(A^{-1}\right)=R(A)$ and $A^{-1} A u=u$ for all $u \in D(A)$. Also, $A A^{-1} v=v$ for all $v \in D\left(A^{-1}\right)$.

Let us now prove that the equalities

$$
\left(A^{-1} u, v\right)_{n}=\left(u, A^{-1} v\right)_{n}
$$

are fulfilled for all $u, v \in D\left(A^{-1}\right)$ and $n \in N$. Indeed, if $A^{-1} u=x$ and $A^{-1} v=y$, then $u=A x v=A y$. Hence by the symmetry of the operator $A$ we have

$$
\left(A^{-1} u, v\right)_{n}=(x, A y)_{n}=(A x, y)_{n}=\left(u, A^{-1} v\right)_{n}
$$

for all $n \in N$, i.e., $A^{-1}$ is symmetrical.

Further, the fact that the operator $A$ is positive definite implies that for each $n \in N$ there exists $\gamma_{n}>0$ such that

$$
(A u, u)_{n} \geq \gamma_{n}^{2}(u, u)_{n} \text { for all } u \in D(A) .
$$

From the Cauchy-Bunyakovski inequality $(A u, u)_{n} \leq\|A u\|_{n}\|u\|_{n}$ it follows that $\gamma_{n}^{2}(u, u)_{n} \leq\|A u\|_{n}\|u\|_{n}$. Hence for $u \in D(A)$, if $\|u\|_{n}=0$, then in the latter relation we have an equality, and if $\|u\|_{n} \neq 0$, then we have the inequality

$$
\gamma_{n}^{2}\|u\|_{n} \leq\|A u\|_{n}
$$

i.e., $A^{-1} \in L_{0}(E)$.

c) Let $A=A^{*}$. In proving the statement b), it was shown that $A^{-1}$ exists and is symmetrical. Therefore there exists its adjoint operator $\left(A^{-1}\right)^{*}$. It is sufficient to prove that $\left(A^{-1}\right)^{*}=\left(A^{*}\right)^{-1}$, since from the condition it follows that $A^{-1}=\left(A^{*}\right)^{-1}$. If $u \in D(A)$ and $v \in D\left(\left(A^{-1}\right)^{*}\right)$, then for each $n \in N$ we have

$$
(u, v)_{n}=\left(A^{-1} A u, v\right)_{n}=\left(A u,\left(A^{-1}\right)^{*} v\right)_{n}=\left(u, A^{*}\left(A^{-1}\right)^{*} v\right)_{n} .
$$

This means that $\left(A^{-1}\right)^{*} v \in D(A)$ and $A^{*}\left(A^{-1}\right)^{*} v=v$. Analogously, if $u \in$ $D\left(A^{-1}\right)$ and $v \in D\left(A^{*}\right)$, then for each $n \in N$ we have

$$
(u, v)_{n}=\left(A A^{-1} u, v\right)_{n}=\left(A^{-1} u, A^{*} v\right)_{n}=\left(u,\left(A^{-1}\right)^{*} A^{*} v\right)_{n},
$$


i.e., $A^{*} v \in D\left(A^{-1}\right)^{*}$ and $\left(A^{-1}\right)^{*} A^{*} v=v$. Hence it follows that $\left(A^{-1}\right)^{*}=$ $\left(A^{*}\right)^{-1}$. The converse statement is proved by applying to $A^{-1}$ the already proven statement.

d) By virtue of the statement b) there exists a symmetrical inverse operator $A^{-1}$. Keeping in mind that $D\left(A^{-1}\right)=R(A)=E$ and by virtue of a), we observe that the operator $A^{-1}$ is selfadjoint, i.e., $A^{-1}=\left(A^{-1}\right)^{*}$. Then, by virtue of c), $A=A^{*}$ and $A$ is selfadjoint.

Note that the statement a) is a generalization of the well-known HellingerToeplitz theorem on the continuity of a symmetrical operator defined on the whole space.

Using Theorem 1, we prove our next generalization of the well known Friedrichs-Stone-Wintner theorem [14].

Theorem 2. Let $E$ be a Fréchet space with a generating increasing sequence of Hilbertian seminorms $\left\{\|\cdot\|_{n}\right\}$ and $A$ be a symmetrical positive definite operator with the dense image $R(A)$. Then $A$ possesses a selfadjoint and positive definite extension $\widetilde{A}$ such that $R(\widetilde{A})=E$.

Proof. By virtue of the proposition b) of Theorem 1 there exists a symmetrical inverse operator $A^{-1}$ to the operator $A$ and $A^{-1} \in L_{0}(E)$. The operator $C=A^{-1}$ can be continuously extended uniquely to the whole space $E$. We denote this extension by $\widetilde{C}$. For all $x \in E$ it is defined by the equality $\widetilde{C} x=\lim _{k \rightarrow \infty} A^{-1} x_{k}$, where $x_{k} \in R(A)$ and $\lim _{k \rightarrow \infty} x_{k}=x$.

Let us prove that $\widetilde{C}$ is also symmetrical. Indeed, let $x, y \in E$ and $\lim _{k \rightarrow \infty} x_{k}=x$, $\lim _{m \rightarrow \infty} y_{m}=y$, where $x_{k}, y_{m} \in R(A)$.

Then for each $n \in N$ we have

$$
\begin{aligned}
& (\widetilde{C} x, y)_{n}=\left(\lim _{k \rightarrow \infty} A^{-1} x_{k}, \lim _{m \rightarrow \infty} y_{m}\right)_{n}=\lim _{k \rightarrow \infty} \lim _{m \rightarrow \infty}\left(A^{-1} x_{k}, y_{m}\right)_{n} \\
= & \lim _{k \rightarrow \infty} \lim _{m \rightarrow \infty}\left(x_{k}, A^{-1} y_{m}\right)_{n}=\lim _{m \rightarrow \infty} \lim _{k \rightarrow \infty}\left(x_{k}, A^{-1} y_{m}\right)_{n}=(x, \widetilde{C} y)_{n} .
\end{aligned}
$$

From the proposition a) of Theorem 1 it follows that $\widetilde{C}$ is selfadjoint. Since $\widetilde{C}$ is the extension of $A^{-1}$, we have $R(\widetilde{C}) \supset R\left(A^{-1}\right)=D(A)$, i.e., $R(\widetilde{C})$ is everywhere dense in $E$. If we now apply the proposition c) of Theorem 1 to the operator $\widetilde{C}$, we establish that $\widetilde{C}^{-1}$ is also selfadjoint. It is shown that $\widetilde{C}^{-1}$ is the selfadjoint extension of the operator $A$. Denote $\widetilde{C}^{-1}$ by $\widetilde{A}$. Let us show that if $x \in D(A)$, then $\widetilde{A} x=A x$. Indeed, then $A x \in R(A)$ and $A^{-1} A x=C A x=x$, i.e., $A x=C^{-1} x$ and therefore $\widetilde{A} x=A x$. Since $R(\widetilde{A})=D(\widetilde{C})=E, \widetilde{A}$ satisfies all the requirements of Theorem 2 .

Corollary. Let E be a Fréchet space with a generating sequence of Hilbertian seminorms $\left\{\|\cdot\|_{n}\right\}$ and $A: E \rightarrow E$ be a symmetrical and positive definite operator with the dense image $R(A)$. Then $A$ possesses an extension $\tilde{A}$ such that the equation $\widetilde{A} u=f$ has a unique solution for each $f \in E$. 
It remains unknown whether Theorem 2 holds without the requirement $\overline{R(A)}=E$. In the case of Hilbert spaces this follows from the fact that the operator $A$ is positive definite ([9], Theorem X.26, p. 205)

Let us show that the solution of the equation $A u=f$ belongs to the energetic space of the operator $A$ which is now defined like in the classical case.

Let $(E, \tau)$ be a Fréchet space with a generating sequence of Hilbertian seminorms $\left\{\|\cdot\|_{n}\right\}$ and $A: E \rightarrow E$ be a symmetrical and positive definite operator. We equip $D(A)$ both with the induced topology $\tau \cap D(A)$ and the topology $\tau_{A}$ generated by the sequence of Hilbertian seminorms

$$
[x]_{n}=[x, x]_{n}^{1 / 2}, \quad x \in D(A), \quad n \in N,
$$

where

$$
[x, y]_{n}=(A x, y)_{n}, \quad x, y \in D(A), \quad n \in N .
$$

From the fact that the operator $A$ is positive definite it follows that the topology $\tau_{A}$ is not weaker than the topology $\tau$. We denote by $E_{A}$ the completion of $D(A)$ in the topology $\tau_{A}$ and call it the energetic space of $A$. The values $[x, y]_{n}$ and $[x]_{n}$ are called energetic products and energetic seminorms, respectively. In [4], the energetic space $E_{A}$ is represented as a projective limit of the sequence of energetic spaces $H_{A_{n}}$ of projection operators $A_{n}$ which are the completion $E / \operatorname{Ker}\|\cdot\|_{n}$ with respect to the norm

$$
\left[K_{n} x\right]_{A_{n}}=\left\langle A_{n} K_{n} x, K_{n} x\right\rangle_{n}, \quad K_{n} x \in D\left(A_{n}\right) .
$$

Since we need only the particular case of this theorem, we will not prove it in a general form.

\section{Selfadjointness of the Restriction to the Fréchet space} $D\left(A^{\infty}\right)$ of the Selfadjoint Operator $A$ in the Hilbert space $H$

Let $H$ be the Hilbert space and $A$ be the selfadjoint operator with a purely pointwise spectrum from $H$ into $H$. According to $([8], \S 5.6 .1), A$ is called an operator with a purely pointwise spectrum if its spectrum consists of isolated points of finite multiplicity and has no finite limiting points.

The topology of the well known countable-Hilbert space $\left.D\left(A^{\infty}\right)=\bigcap_{k=1}^{\infty} D\left(A^{k-1}\right)\right)$ can be given by the following sequence of Hilbertian norms

$$
\|x\|_{n}=\left(\|x\|^{2}+\|A x\|^{2}+\cdots+\left\|A^{n-1} x\right\|^{2}\right)^{1 / 2}, \quad x \in D(A), \quad n \in N,
$$

that are generated by scalar products

$$
(x, y)_{n}=(x, y)+(A x, A y)+\cdots+\left(A^{n-1} x, A^{n-1} y\right), \quad x, y \in D\left(A^{\infty}\right) .
$$

The space $D\left(A^{\infty}\right)$ coincides with the space $H$ if and only if the operator $A$ is bounded. Also note that the sequence $\left\{h_{k}\right\}$ converges to $h$ in the space $D\left(A^{\infty}\right)$ if and only if $A^{n} h_{k}$ converges to $A^{n} h$ in $H$ for each $n \in N$.

In [7], the conditions are given in terms of distribution of the modules of eigenvalues of the operator $A$ on the straight line, under which the space $D\left(A^{\infty}\right)$ is Montel, nuclear or isomorphic to the space of quickly decreasing sequences $s$. 
It is well known that each Fréchet space is isomorphic to a subspace of the product of sequences of Banach spaces. The space $D\left(A^{\infty}\right)$ is isomorphic to a subspace $M$ of the Fréchet-Hilbert space $H^{N}$ [1], the topology of which is generated by the following sequence of semiscalar products

$$
(x, y)_{n}=\left(x_{1}, y_{1}\right)+\cdots+\left(x_{n}, y_{n}\right), \quad x=\left\{x_{k}\right\}, \quad y=\left\{y_{k}\right\} \in H^{N}, \quad n \in N .
$$

The above-mentioned isomorphism (it actually is an isometry) is obtained by the mapping

$$
D\left(A^{\infty}\right) \ni x \rightarrow \operatorname{Orb}(A, x):=\left\{x, A x, \ldots, A^{n-1} x, \ldots\right\} \in M \subset H^{N} .
$$

Therefore the space $D\left(A^{\infty}\right)$ is isomorphic to the space of all orbits $\operatorname{orb}(A, x)$ for the operator $A$ at a point $x \in D\left(A^{\infty}\right)$ considered in the induced topology of the space $H^{N}$. Using this representation we define the operator $A^{\infty}: D\left(A^{\infty}\right) \rightarrow$ $D\left(A^{\infty}\right)$ by the equality

$$
A^{\infty} x=A^{\infty}\left\{x, A x, A^{2} x, \ldots\right\}=\left\{A x, A^{2} x, \ldots\right\} .
$$

Actually, $A^{\infty}$ is the restriction on $D\left(A^{\infty}\right)$ of the operator $A^{N}$ defined on $H^{N}$ by the equality

$$
A^{N}\left(\left\{x_{k}\right\}\right)=\left\{A x_{k}\right\} \in H^{N} .
$$

Theorem 3. Let $A$ be the selfadjoint operator in the Hilbert space $H$ with the dense domain $D(A)$. Then the following propositions are true:

a) The operator $A^{N}$ is a selfadjoint operator in the Fréchet-Hilbert space $H^{N}$ with a sequence of Hilbertian seminorms (9) and the domain $D(A)^{N}$.

b) The operator $A^{\infty}$, defined by equality (10) on the whole space $D\left(A^{\infty}\right)$ is a continuous selfadjoint operator in the space $D\left(A^{\infty}\right)$.

c) If the operator $A$ has a purely pointwise spectrum, then $A^{\infty}$ possesses a selfadjoint inverse operator $\left(A^{\infty}\right)^{-1}$ in the space $D\left(A^{\infty}\right)$. Further, if $A$ is positive definite in $H$, then $A^{\infty}$ is an isomorphism of the space $D\left(A^{\infty}\right)$ onto and the energetic space $E_{A^{\infty}}$ of the operator $A^{\infty}$ coincides with $D\left(A^{\infty}\right)$.

Proof. a) It is not difficult to show that the Hilbert adjoint operator of $A^{N}$ in the Fréchet-Hilbert space $H^{N}$ is $\left(A^{*}\right)^{N}$ with the domain $D\left(A^{*}\right)^{N}$ and therefore the condition implies that the operator $A^{N}$ is selfadjoint in $H^{N}$.

b) It is obvious that the definition domain of the operator $A^{\infty}$ is the whole space $D\left(A^{\infty}\right)$, i.e., from now on the notation of the space $D\left(A^{\infty}\right)$ is also the notation of the definition domain of the operator $A^{\infty}$ (which is natural). Let $x, y \in D\left(A^{\infty}\right)$, then for each $n \in N$ we have

$$
\begin{aligned}
& \left(A^{\infty} x, y\right)_{n}=(A x, y)+\left(A^{2} x, A y\right)+\cdots+\left(A^{n} x, A^{n-1} y\right) \\
= & (x, A y)+\left(A x, A^{2} y\right)+\cdots+\left(A^{n-1} x, A^{n} y\right)=\left(x, A^{\infty} y\right)_{n} .
\end{aligned}
$$

Hence, by virtue of the proposition a) of Theorem 1 , we obtain that $A^{\infty}$ is a continuous selfadjoint operator in the space $D\left(A^{\infty}\right)$.

c) By Rellich's theorem ([8], §5.6.1) the selfadjoint operator in the Hilbert space $H$ has a purely pointwise spectrum if and only if the embedding of the definition domain $D(A)$ (with the norm $\|u\|_{D(A)}=\|A u\|+\|u\|$ ) into $H$ is compact. Hence it follows that the space $D\left(A^{\infty}\right)$ is a space of the type $(F S)$, i.e., 
is a projective limit of a sequence of Hilbert spaces with compact embeddings. In particular, in our case the space $D\left(A^{\infty}\right)$ is Montel, i.e., each closed bounded set in it is compact. By virtue of Theorem 3 from [15], the operator $A$ possesses a complete system of orthonormalized in $H$ eigenelements $\left\{\varphi_{k}\right\}$ (with eigenvalues $\lambda_{k}$ ) which is at the same time an unconditional basis in $D\left(A^{\infty}\right)$. (If the space $D\left(A^{\infty}\right)$ is nuclear, then this basis is absolute). Hence it follows that the image $R\left(A^{\infty}\right)$ of the operator $A^{\infty}$ is everywhere dense in the space $D\left(A^{\infty}\right)$, since each eigenelement of the operator $A$ is also an eigenelement for the operator $A^{\infty}$ and is contained in its image. Indeed, by virtue of the above-mentioned isomorphism, for any $k \in N$ we have

$$
\begin{gathered}
A^{\infty} \varphi_{k}=A^{\infty}\left(\varphi_{k}, A \varphi_{k}, A^{2} \varphi_{k}, \ldots\right)=\left(A \varphi_{k}, A^{2} \varphi_{k}, A^{3} \varphi_{k}, \ldots\right) \\
=\left(\lambda_{k} \varphi_{k}, \lambda_{k} A \varphi_{k}, \lambda_{k} A^{2} \varphi_{k}, \ldots\right)=\lambda_{k}\left(\varphi_{k}, A \varphi_{k}, A^{2} \varphi_{k}, \ldots\right)=\lambda_{k} \varphi_{k} .
\end{gathered}
$$

Therefore, by the proposition c) of Theorem 1, we conclude that $A^{\infty}$ possesses a selfadjoint inverse operator $\left(A^{\infty}\right)^{-1}$.

Let now $A$ be a positive definite operator in $H$, then for $x \in D\left(A^{\infty}\right)$ and $n \in N$ we have

$$
\begin{gathered}
\left(A^{\infty} x, x\right)_{n}=(A x, x)+\left(A^{2} x, A x\right)+\cdots+\left(A^{n} x, A^{n-1} x\right) \\
\geq \gamma\left[(x, x)+(A x, A x)+\cdots+\left(A^{n-1} x, A^{n-1} x\right)\right]=\gamma^{2}(x, x)_{n},
\end{gathered}
$$

i.e., $A^{\infty}$ is also positive definite in $D\left(A^{\infty}\right)$. By virtue of the proposition b) of Theorem $1\left(A^{\infty}\right)^{-1} \in L_{0}\left(D\left(A^{\infty}\right)\right)$. Since by Theorem $2\left(A^{\infty}\right)^{-1}$ possesses

a selfadjoint extension $\widetilde{\left(A^{\infty}\right)^{-1}}$ such that $D\left(\widetilde{\left(A^{\infty}\right)^{-1}}\right)=D\left(A^{\infty}\right)$, we obtain $\widetilde{\left(A^{\infty}\right)^{-1}}=\left(A^{\infty}\right)^{-1}$. Therefore $A^{\infty}$ is an isomorphism of the space $D\left(A^{\infty}\right)$ onto.

The energetic space $E_{A^{\infty}}$ of the operator $A^{\infty}$ is considered using the topology $\tau_{A^{\infty}}$ which is generated by the sequence of norms (6) and has the form

$$
[x]_{n}^{2}=\left(A^{\infty} x, x\right)_{n}=(A x, x)+\cdots+\left(A^{n} x, A^{n-1} x\right) .
$$

By virtue of (11) we have $[x]_{n}^{2} \geq \gamma^{2}(x, x)_{n}$. Thus the topology $\tau_{A^{\infty}}$ is not weaker than the topology of the space $D\left(A^{\infty}\right)$ and therefore these topologies coincide, i.e., $E_{A^{\infty}}=D\left(A^{\infty}\right)$. In particular, this means that the equation $A^{\infty} u=f$ has a unique, strongly stable solution in $D\left(A^{\infty}\right)$ for any $f \in D\left(A^{\infty}\right)$.

\section{Application of the Extended Ritz Method for Obtaining an Approximate Solution of the Equation with a Strongly Degenerating Elliptical Operator}

To obtain an approximate solution of equation (2) in the space $D\left(A^{\infty}\right)$ we apply the extension of the Ritz method from [4] (approximation methods of the solution of operator equations in Fréchet spaces were previously considered in [18]-[19]). Since we need only the particular case of this method, we do not give its proof in the general case. As basic functions we choose a sequence of eigenfunctions $\left\{\varphi_{j}\right\}$ of the operators $A$ and $A^{\infty}$. For $l \in N$, a system of 
equations for defining the coefficients of an approximate solution is written in the form

$$
\sum_{k=1}^{m}\left[\varphi_{k}, \varphi_{j}\right]_{l} \alpha_{k}=\left(f, \varphi_{j}\right)_{l}, \quad j=1, \ldots, m
$$

i.e.,

$$
\sum_{k=1}^{m}\left(A^{\infty} \varphi_{k}, \varphi_{j}\right)_{l} a_{k}=\left(f, \varphi_{j}\right)_{l}, \quad j=1, \ldots, m
$$

where

$$
\begin{gathered}
{\left[\varphi_{k}, \varphi_{j}\right]_{l}=\left(A^{\infty} \varphi_{k}, \varphi_{j}\right)_{l}=\left(A \varphi_{k}, \varphi_{j}\right)+\left(A^{2} \varphi_{k}, A \varphi_{j}\right)+\cdots+\left(A^{l} \varphi_{k}, A^{l-1} \varphi_{j}\right)} \\
=\left(\lambda_{k}+\lambda_{k}^{2} \lambda_{j}+\cdots+\lambda_{k}^{l} \lambda_{j}^{1-1}\right)\left(\varphi_{k}, \varphi_{j}\right) .
\end{gathered}
$$

Moreover, we have

$$
\begin{aligned}
& \left(f, \varphi_{j}\right)_{l}=\left(f, \varphi_{j}\right)+\left(A f, A \varphi_{j}\right)+\cdots+\left(A^{l-1} f, A^{l-1} \varphi_{j}\right)=\left(f, \varphi_{j}\right) \\
& +\lambda_{j}^{2}\left(f, \varphi_{j}\right)+\cdots+\lambda_{j}^{2(l-1)}\left(f, \varphi_{j}\right)=\left(1+\lambda_{j}^{2}+\cdots+\lambda_{j}^{2(l-1)}\right)\left(f, \varphi_{j}\right) .
\end{aligned}
$$

If the sequence $\left\{\varphi_{j}\right\}$ is orthogonal in $H$, then from (14) we obtain

$$
\left[\varphi_{k}, \varphi_{j}\right]_{l}=\left\{\begin{array}{l}
0, \text { when } k \neq j, \\
{\left[\varphi_{j}\right]_{l}^{2}=\left(\lambda_{j}+\lambda_{j}^{3}+\cdots+\lambda_{j}{ }^{2 l-1}\right), \quad \text { when } k=j}
\end{array}\right.
$$

Hence it follows that

$$
\alpha_{j}=\frac{\left(1+\lambda_{j}^{2}+\cdots+\lambda_{J}^{2(l-1)}\right)\left(f, \varphi_{j}\right)}{\lambda_{j}\left(1+\lambda_{j}^{2}+\cdots+\lambda_{J}^{2(1-1)}\right)\left\|\varphi_{j}\right\|^{2}}=\frac{\left(f, \varphi_{j}\right)}{\lambda_{j}\left\|\varphi_{j}\right\|^{2}} .
$$

Therefore an approximate solution takes the form

$$
u_{m}=\sum_{j=1}^{m} \frac{\left(f, \varphi_{j}\right)}{\lambda_{j}\left\|\varphi_{j}\right\|^{2}} \varphi_{j}
$$

If the sequence $\left\{\varphi_{j}\right\}$ is orthonormalized in $H$, then

$$
u_{m}=\sum_{j=1}^{m} \frac{\left(f, \varphi_{j}\right)}{\lambda_{j}} \varphi_{j}
$$

Note that approximate solutions (16) do not depend on $l \in N$. Therefore, by virtue of the classical Ritz theorem, this sequence converges to a solution of equation (2) with respect to energetic norms $[\cdot]_{n}(12)$. Indeed, in that case the canonical mappings $K_{A^{\infty}, n}: E_{A^{\infty}} \rightarrow E_{A^{\infty}} / \operatorname{Ker}[\cdot]_{n}$ are the identical mappings $J_{n}: D\left(A^{\infty}\right) \rightarrow\left(D\left(A^{\infty}\right),[\cdot]_{n}\right)$ defined by the equality

$$
J_{n} x=J_{n}(x, A x, \ldots)=\left(x, A x, \ldots, A^{n-1} x\right),
$$

while the projection operators (5) written as

$$
A_{n}^{\infty}\left(J_{n} x\right)=J_{n}\left(A^{\infty} x\right)=J_{n}\left(\left\{A x, A^{2} x, \ldots,\right\}\right)=\left(A x, A^{2} x, \ldots, A^{n} x\right)
$$


are the operators considered in Hilbert spaces $\left(\widetilde{D\left(A^{\infty}\right)},[\cdot]_{n}\right)$, where $\left.\left(\widetilde{D\left(A^{\infty}\right.}\right),[\cdot]_{n}\right)$ is the completion of the normed space $\left(D\left(A^{\infty}\right),[\cdot]_{n}\right)$. But the norms $[\cdot]_{n}$ are isometric to the norms $[\cdot]_{A_{n}^{\infty}}$ for each $n \in N$, where

$$
\left[J_{n} x\right]_{A_{n}^{\infty}}=\left\langle A_{n}^{\infty} J_{n} x, J_{n} x\right\rangle_{n}^{1 / 2}=\left((A x, x)+\cdots+\left(A^{n} x, A^{n-1} x\right)\right)^{1 / 2} .
$$

Therefore the sequence $\left\{u_{m}\right\}$ converges to $\left(A^{\infty}\right)^{-1} f$ in the energetic space $E_{A^{\infty}}$ and, accordingly, in the space $D\left(A^{\infty}\right)$. We have thereby proved that the following statement is true.

Theorem 4. Let $A$ be a selfadjoint positive definite operator in the Hilbert space $H$ with an orthogonal sequence of eigenfunctions $\left\{\varphi_{j}\right\}$. Then the sequence of approximate solutions $\left\{u_{m}\right\}$ constructed by the extended Ritz method (15) converges to a solution of equation (2) in the space $D\left(A^{\infty}\right)$.

Now we will give the definitions of a generalized spline algorithm and a generalized central algorithm which are respectively a generalization of the spline algorithm and the central algorithm from [20]. Further, it will be shown that the algorithm costructed in Theorem 4 for an approximate solution of equation (2) in the space $D\left(A^{\infty}\right)$ is simultaneously a generalized central and a generalized spline algorithm. For the metrization of the space $D\left(A^{\infty}\right)$ we use the metric from [19] constructed for an increasing sequence of norms $\left\{[\cdot]_{n}\right\}$. For an arbitrary Fréchet space with an increasing sequence of seminorms $\left\{\|\cdot\|_{n}\right\}$ this metric has the form

$$
d(x, y)=\left\{\begin{array}{l}
\|x-y\|_{1}, \text { when }\|x-y\|_{1} \geq 1 \\
2^{-n+1}, \text { when }\|x-y\|_{n} \leq 2^{-n+1} \text { and } \\
\|x-y\|_{n+1} \geq 2^{-n+1}(n \in N), \\
\|x-y\|_{n+1}, \text { when } 2^{-n} \leq\|x-y\|_{n+1}<2^{-n+1} \quad(n \in N), \\
0, \text { when } x-y=0 .
\end{array}\right.
$$

Let $F_{1}$ be a linear space, $G$ be the normed space over the scalar field of real or complex numbers and $S: F_{1} \rightarrow G$ be a linear operator. Let $F$ be a balanced convex subset of the space $F_{1}$. Let $U(f)$ be the computed approximation of an element $S(f)$. To compute $U(f)$ we need to have certain knowledge about the elements $f \in F$. Let $y=I(f)=\left[L_{1}(f), \ldots, L_{m}(f)\right]$ be the computed nonadaptive information with cardinality $m$ (as different from [20] we denote this infomartion by $I$ ), where $L_{1}, \ldots, L_{m}$ are linear functionals on $F_{1}$. If $y=$ $I(f)$ is known, then the approximation of $U(f)$ computed by means of this information gives an element of the space $G$ which is the approximation of the element $S(f)$. Therefore $U(f)=\varphi(I(f))$, where $\varphi: I\left(F_{1}\right) \rightarrow G$ is the mapping called the algorithm.

The local error for $U=(\varphi, I)$ is defined as follows: given $y=I(f)$, the element $\varphi(y)$ has to approximate all elements from the set $S\left(I^{-1} y\right)$, where $S\left(I^{-1} y\right)=\left\{S(f), f \in I^{-1}(y)\right\}$ and $I^{-1}(y)=\{f \in F ; I(f)=y\}$. The value

$$
e(\varphi, I, y)=\sup \left\{\left\|S\left(f^{\prime}\right)-\varphi(y)\right\| ; f^{\prime} \in I^{-1}(y)\right\}
$$

is called the local error. 
The radius of the set $S\left(I^{-1}(y)\right)$ is denoted by $r(I, y)$ and called the local radius of the information $I$ at a point $y$. Let the set $S\left(I^{-1}(y)\right)$ have the Chebyshev center $\mathbf{m}=\mathbf{m}(y)$ for each $y \in I(F)$. Then the algorithm $\varphi^{c}(y)=\mathbf{m}(y)$ is called central. For the central algorithm we have

$$
\begin{gathered}
r(I, y)=\operatorname{rad} S\left(I^{-1}(y)\right)=\inf \left\{\sup \left\{\left\|S\left(f^{\prime}\right)-g\right\| ; f^{\prime} \in I^{-1}(y)\right\} ; g \in G\right\} \\
=\sup \left\{\left\|S\left(f^{\prime}\right)-\mathbf{m}(y)\right\| ; f^{\prime} \in I^{-1}(y)\right\}=e\left(\varphi^{c}, I, y\right),
\end{gathered}
$$

i.e., the local error of the central algorithm coincides with the local radius and is therefore minimal.

Sometimes it is convenient to assume that the set $F$ is generated by the restriction operator $T: F_{1} \rightarrow X$, where $\left(X,\|\cdot\|_{F}\right)$ is the normed space and $F=\left\{f \in F_{1} ;\|T(f)\|_{F} \leq 1\right\}$. Indeed, the space $X$ coincides with Ker $\mu_{F}^{\perp}$, where $\mu_{F}$ is the Minkovski functional for the set $F$, Ker $\mu_{F}^{\perp}$ is an algebraic complement of a subset $\operatorname{Ker} \mu_{F}, T: F_{1} \rightarrow \operatorname{Ker} \mu_{F}^{\perp}$ an algebraic projector, and the norm $\|\cdot\|_{X}$ on $X$ is defined by the equality $\|T f\|_{X}=\mu_{F}(f)$.

$\sigma=\sigma(y)$ is called the interpolation spline for $y \in I\left(F_{1}\right)$ if $I(\sigma)=y$ and

$$
\|T \sigma\|=\min \left\{\|T z\| ; \quad z \in F_{1}, I(z)=y\right\} .
$$

According to [12], for the above-mentioned mapping $T$, the nonadaptive information $I$ and each $y \in I\left(F_{1}\right)$, the interpolation spline exists if and only if the subspace Ker $I$ is proximinal in $F_{1}$ with respect to $\mu_{F}$.

If the interpolation spline exists and is unique, then the spline algorithm is defined by the equality

$$
\Phi^{s}(y)=S(\sigma(y)), \quad y \in I\left(F_{1}\right),
$$

where $\sigma(y)$ is the interpolation spline for $y$.

Using the result from [12], we will now give the definition of a generalized spline in terms of best approximations. Let $F_{1}$ be a metrizable, locally convex space on which there exists a metric $d$, invariant with respect to translation and having absolutely convex balls $K_{r}=\left\{x \in F_{1} ; d(x, 0) \leq r\right\}$, such that $\left(F_{1}, d\right)$ is a metric linear space. Denote by $q_{r}(\cdot)$ the Minkowski functional of a ball $K_{r}$, and by $|\cdot|$ the quasinorm of the metric $d$. Let, further, $I$ be again the nonadaptive information of cardinality $m, y \in I\left(F_{1}\right), I(f)=y$ for some $f \in F_{1}$ and $d(f, \operatorname{Ker} I)=r$. Then $\sigma=f-h^{*}$ is called the generalized interpolation spline if $I(\sigma)=y$,

$$
d(f, \operatorname{Ker} I)=d\left(f, h^{*}\right)=r=d(\sigma, 0)=|\sigma|
$$

and

$$
\inf \left\{q_{r}(f-h) ; h \in \operatorname{Ker} I\right\}=q_{r}\left(f-h^{*}\right)=q_{r}(\sigma),
$$

i.e., the generalized spline $\sigma$ minimizes not only the metric but also the corresponding Minkowski functional.

Note that this definition does not depend on the choice of an element $f \in F_{1}$ for which $I(f)=y$ and $d(f, \operatorname{Ker} I)=r$. Indeed, since if

$$
I\left(f_{1}\right)=I\left(f_{2}\right)=y, \quad f_{2}-f_{1}=z \in \operatorname{Ker} I, \quad d\left(f_{1}, \operatorname{Ker} I\right)=d\left(f_{1}, h_{1}^{*}\right),
$$


and

$$
\left.\inf \left\{q_{r}\left(f_{1}-h\right) ; h \in \operatorname{Ker} I\right)\right\}=q_{r}\left(f_{1}-h_{1}^{*}\right)
$$

then

$$
d\left(f_{2}, \operatorname{Ker} I\right)=d\left(f_{2}, h_{1}^{*}+z\right)=d\left(f_{1}, h_{1}^{*}\right)
$$

and

$$
\inf \left\{q_{r}\left(f_{2}-h\right) ; h \in \operatorname{Ker} I\right\}=q_{r}\left(f_{2}-h_{1}^{*}+z\right)=q_{r}\left(f_{1}-h_{1}^{*}\right) .
$$

By virtue of the results obtained in [21] for metric (17), in terms of the above notation $\sigma=f-h^{*}$ is a generalized interpolation spline if and only if $I(\sigma)=y$,

$$
d(f, \operatorname{Ker} I)=d\left(f, h^{*}\right)=r \neq 2^{-n+1} \quad(n \in N)
$$

and

$$
\begin{gathered}
E\left(f, \operatorname{Ker} I, V_{n}\right):=\inf \left\{\|f-h\|_{n} ; h \in \operatorname{Ker} I\right\}=\left\|f-h^{*}\right\|_{n}, \\
\text { when } \quad r=2^{-n+1} \quad(n \in N),
\end{gathered}
$$

where $V_{n}=\left\{x \in F_{1} ;\|x\|_{n} \leq 1\right\}$ and $\left\{\|\cdot\|_{n}\right\}$ is an increasing sequence of seminorms that participates in the definition of the metric. For $V_{1}=V_{2}=$ $\cdots=F$ we prove that $K_{r}=r F,|\cdot|=\mu_{F}(\cdot)$ and the generalized interpolation spline coincides with the classical one.

We use the fact that the Minkowski functionals $q_{r}(\cdot)$ of the balls $K_{r}$ of this metric have the form $q_{r}(x)=r^{-1}\|\cdot\|_{n}$, where

$$
r \in I_{n}= \begin{cases}{[1, \infty[} & \text { for } n=1 \\ {\left[2^{-n+1}, 2^{-n+2}[\right.} & \text { for } n \geq 2 .\end{cases}
$$

When the generalized spline exists and is unique, then the generalized spline algorithm is also defined by equality (18).

It should be noted that the existence of a generalized spline in the case of nonadaptive information of cardinality 1 in terms of the proximality of closed hypersubspaces was considered by many mathematicians and the final results were obtained in [21].

Let us now define the notion of a generalized central algorithm in the case of metric (17).

Let $I$ be nonadaptive information of cardinality $m \geq 1, y \in I\left(F_{1}\right)=R^{m}$, $I(f)=y$ and $d(f$, Ker $I)=r \in I_{n}$ for some $f \in F_{1}$. We call the value

$$
e(\varphi, I, y)=\sup \left\{\|S(f)-\varphi(y)\| ; f \in I^{-1}(y) \cap V_{n}\right\},
$$

the local error of the algorithm $\varphi$ at a point $y$.

Denote by $r(I, y)$ the local radius of the nonadaptive information $I$ at a point $y$ defined by the equality

$$
r(I, y)=\operatorname{rad}\left(S\left(I^{-1}(y) \cap V_{n}\right)\right) .
$$

Let for each $y$ there exists a Chebyshev center $\mathbf{m}(y)$ of the set $S\left(I^{-1}(y) \cap V_{n}\right)$. Then the algorithm $\varphi^{c}$ is defined by the equality

$$
\varphi^{c}(y)=\mathbf{m}(y), \quad y \in R^{m},
$$


and called generalized central. As has been noted above, if $V_{1}=V_{2}=\cdots=F$, then the notion of generalized centrality coincides with the classical definition.

Denote by $r_{n}(I)$ the global radius of nonadaptive information defined by the equality

$$
r_{n}(I)=\sup \left\{r(I, y) ; y \in I\left(V_{n}\right)\right\} .
$$

Assume that the topology of the Fréchet space $E$ is given by a sequence of Hilbert seminorms $\left\{\|\cdot\|_{n}\right\}$, i.e., each seminorm $\|\cdot\|_{n}$ is generated by the semiscalar product $(x, y)_{n}$ and $V_{n}=\left\{x \in E ;\|x\|_{n} \leq 1\right\}$. For such spaces the notion of orthogonality is naturally defined as follows: elements $x, y \in E$ are called orthogonal if $(x, y)_{n}=0$ for each $n \in N$. A subspace $M$ possesses an orthogonal complement $M^{\perp}$ in $E$ if each element $x \in E$ is represented as a sum $x=y+z$, where $y \in M, z \in M^{\perp}$ and $(y, z)_{n}=0$ for each $n \in N$. In other words, this means that in subspaces $M$ and $M^{\perp}$ each element $x \in E$ possesses a unique best approximation $y$ and $z$, respectively, with respect to all seminorms $\|\cdot\|_{n}$ generated by $(\cdot, \cdot)_{n}$.

Theorem 5. Let $E=F_{1}$ be a Fréchet space with a decreasing sequence of absolutely convex neighborhoods of zero $V_{n}=\left\{x \in E ;\|x\|_{n} \leq 1\right\}$ for which seminorms $\|\cdot\|_{n}$ are generated by semiscalar products and metric $(17), K_{n}$ be the canonical mapping $F_{1} \rightarrow F_{1} /$ Ker $\|\cdot\|_{n}$, $G$ be the normed space, $S: F_{1} \rightarrow G$ be a linear operator, $I$ be nonadaptive information of cardinality $m \geq 1$ and $r_{1}(I)<\infty$. Then if the subspace Ker I possesses an orthogonal complement in $F_{1}$ and $K_{n}(\operatorname{Ker} I)$ is closed in $\left(F_{1} /\right.$ Ker $\left.\|\cdot\|_{n},\|\cdot\|_{n}\right)$, then there exists a generalized spline algorithm $\Phi^{s}$ which is linear and generalized central.

Proof. Let $I$ be nonadaptive information of cardinality $m \geq 1, y \in I\left(F_{1}\right)=R^{m}$, $I(f)=y$ and $d(f, \operatorname{Ker} I)=r \in I_{n}$ for some $f \in F_{1}$. Since the subspace Ker $I$ possesses an orthogonal complement in $F_{1}$, for each $f \in F_{1}$ there exist $h \in \operatorname{Ker} I$ and $\sigma \in \operatorname{Ker} I^{\perp}$ such that $f=h+\sigma$ and $(h, \sigma)_{n}=0$ for each $n \in N$, where $(\cdot, \cdot)_{n}$ is a semiscalar product generating a seminorm $\|\cdot\|_{n}$. This means that $h$ and $\sigma$ are elements of the best approximation of $f$ in the subspaces Ker $I$ and Ker $I^{\perp}$ and with respect to metric (17). Therefore $\sigma$ is a generalized spline and the correspondence $y \rightarrow \sigma$ is linear. Thus the spline algorithm $\Phi^{s}$ is linear.

It remains to prove that $\Phi^{s}$ is generalized central, i.e., that the set $S\left(I^{-1}(y) \cap\right.$ $V_{n}$ ) has a Chebyshev center for each $y \in R^{m}$. By virtue of $[20$, p. 50] this statement follows from the fact that the set $I^{-1}(y) \cap V_{n}$ is symmetrical with respect to $\sigma$ ([20], p. 97) and therefore the set $S\left(I^{-1}(y) \cap V_{n}\right)$ is symmetrical with respect to $\Phi^{s}(y)=S(\sigma)$.

According to [12], the condition $r_{1}(I)<\infty$ is equivalent to the inequality

$$
\|S\|_{\text {Ker } I}=\sup \left\{\|S(h)\| ; h \in \operatorname{Ker} I,\|h\|_{1} \leq 1\right\}<\infty .
$$

Let $H$ be the Hilbert space with a scalar product $(\cdot, \cdot), S$ be a selfadjoint positive definite operator from $H$ into $H$ with a sequence of eigenvectors $\left\{\varphi_{i}\right\}$, and $F_{1}$ be the Fréchet space $D\left(S^{\infty}\right)$ with the sequence of Hilbertian norms

$$
[x]_{n}^{2}=\left(S^{\infty} x, x\right)_{n}=(S x, x)+\left(S^{2} x, S x\right)+\cdots+\left(S^{n} x, S^{n-1} x\right),
$$


where $V_{n}=\left\{x \in D\left(S^{\infty}\right) ;[x]_{n} \leq 1\right\}$, the information If $=\left[\left(S f, \varphi_{1}\right), \ldots\right.$, $\left.\left(S f, \varphi_{m}\right)\right]=\left(y_{1}, \ldots, y_{m}\right)$ and $\operatorname{Ker} I=\left\{x \in D\left(S^{\infty}\right) ;\left[x, \varphi_{1}\right]=0, \ldots,\left[x, \varphi_{m}\right]=0\right\}$. Its orthogonal subspace Ker $I^{\perp}=\operatorname{span}\left\{\varphi_{1}, \ldots, \varphi_{m}\right\}$. Ker $I$ possesses an orthogonal complement in the Fréchet space $D\left(S^{\infty}\right)$, since the best approximation of an element $u \in D\left(S^{\infty}\right)$ in $\operatorname{Ker} I^{\perp}$ does not depend on $n$, and the spline $\sigma$ is the best approximation of an element $u$ in $\operatorname{Ker} I^{\perp}$. Therefore the conditions of Theorem 5 are fulfilled and thus there exists a generalized spline and spline algorithm for the solution of the equation $S^{\infty} u=f$, which is generalized central. In reality, we have the information $\left(y_{1}, \ldots, y_{m}\right)=\left[\left(S u, \varphi_{1}\right), \ldots,\left(S u, \varphi_{m}\right)\right]$ and we find the best approximation of an element $u$ in the subspace Ker $I$, which possesses an orthogonal complement $\operatorname{Ker} I^{\perp}$ spanned over $\varphi_{1}, \ldots, \varphi_{m}$. Hence the spline $\sigma$ is the best approximation for $u$ in Ker $I^{\perp}$ with respect to all energetic norms $[\cdot]_{n}$ and coincides with the approximate solution constructed by the Ritz method.

It will be also noted that an operator $A: F_{1} \rightarrow F_{1} \subset G^{N}$, where $F_{1}$ is a Fréchet space, generates the sequence of linear problems with the solution operator $A_{n}: F_{1} \rightarrow G$ and with the set of problem elements $V_{n}$ ([20], p. 56).

In particular, according to [13] the conditions of Theorem 5 are satisfied by

a) the information $I$ in Fréchet-Hilbert spaces $F_{1}$ for which a subspace Ker $I$ is again a Fréchet-Hilbert space;

b) by the information $I$ in countable-Hilbert spaces generated by continuous functionals of the space $\left(F_{1},\|\cdot\|_{1}\right)^{\prime}$.

We will now give a few examples of selfadjoint and positive definite differential operators in Hilbert spaces, which satisfy the conditions of Theorem 4. These examples are taken mainly from [7] and [8].

For an arbitrary domain $\Omega \subset R^{l}$ we as usual denote by $C^{\infty}(\Omega)$ the space of all infinitely differentiable functions defined in $\Omega$. Further, let $\rho(x) \in C^{\infty}(\Omega)$ be a positive function such that

1. For any multi-index $\gamma$ there exists $C_{\gamma}>0$ such that $\left|D^{\gamma} \rho(x)\right| \leq C_{\gamma} \rho^{1+|\gamma|}(x)$, for all $x \in \Omega$.

2. For any $K>0$ there exist numbers $\varepsilon_{k}>0$ and $r_{k}>0$ such that $\rho(x)>K$ if $d(x) \leq \varepsilon_{k}$ or $|x| \geq r_{k}$ when $x \in \Omega(d(x)$ is a distance from $x$ to the boundary $\partial(\Omega))$.

Denote by $S_{\rho(x)}(\Omega)$ the metrizable, locally convex space

$$
\begin{gathered}
S_{\rho(x)}(\Omega)=\left\{f \in C^{\infty}(\Omega) ; \quad\|f\|_{n, \alpha}=\sup \rho^{n}(x)\left|D^{\alpha} f(x)\right|<\infty,\right. \\
\text { for all } n=0,1, \ldots \text { and all multi-indices } \alpha\} .
\end{gathered}
$$

Note that for each bounded domain $\Omega$ there exists a function $\rho(x)$ for which $\rho^{-1}(x)$ actually coincides with $d(x)$.

As is known $([8], \S 6.2 .3)$, the space $S_{\rho(x)}(\Omega)$ is a Fréchet space that contains $C_{0}^{\infty}(\Omega)$ as a dense subspace. In what follows, we will consider spaces $S_{\rho(x)}(\Omega)$ contained in the space $L^{p}(\Omega)(1 \leq p<\infty)$. This is equivalent to the condition: there exists $a>0$ for which $\rho^{-\bar{a}}(x) \in L^{1}(\Omega)$. By virtue of the theorem ([8], $\S 6.2 .3)$, the space $S_{\rho(x)}(\Omega)$ is a nuclear Fréchet space isomorphic to the space 
of fast decreasing sequences $s$. It will be shown below that the well known Schwartz space $S(R)$ is a particular case of such spaces.

Let $\Omega \subset R^{l}$ be an arbitrary domain and $\rho(x)$ be the above-mentioned weight function. Further, let $r \in N, \mu$ and $\nu$ be real numbers, $\nu>\mu+2 r$. Put

$$
\chi_{q}=\frac{1}{2 r}(\nu(2 r-q)+\mu q), \quad q=0,1, \ldots, 2 r .
$$

The class $N_{\mu, \nu}^{r}(\Omega, \rho(x))$ consists of all differentiable operators of the form

$$
A u=\sum_{q=0}^{m} \sum_{|j|=2 q} \rho^{\chi_{2 q}}(x) b_{\alpha}(x) D^{\alpha} u+\sum_{|\beta|<2 r} \alpha_{\beta}(x) D^{\beta} u .
$$

Here $b_{\alpha}(x) \in C^{\infty}(\Omega) \quad(|\alpha|=2 q$, where $q=0,1, \ldots, r)$ are real functions whose all derivatives of which (including the functions themselves) are bounded in $\Omega$. Besides, it is assumed that there exists a positive number $C$ such that for all $\xi \in R^{n}$ and $x \in \Omega$

$$
\begin{gathered}
(-1)^{r} \sum_{|\alpha|=2 r} b_{\alpha}(x) \xi^{\alpha} \geq C|\xi|^{2 r}, \quad b_{(0, \ldots, 0)}(x) \geq C \\
(-1)^{q} \sum_{|\alpha|=2 q} b_{\alpha}(x) \xi^{\alpha} \geq 0, \quad q=1, \ldots, r-1
\end{gathered}
$$

(ellipticity condition). Finally, $\alpha_{\beta}(x) \in C^{\infty}(\Omega) \quad(0 \leq|\beta|<2 r)$ and $D^{\gamma} \alpha_{\beta}(x)=$ $o\left(\rho^{\chi|\beta|}+|\gamma|\right)$ for any multi-index $\gamma$.

The class $\mathfrak{R}_{\mu, \nu}^{r}(\Omega, \rho(x))$ is quite a wide class of degenerating elliptical differential operators. We will give an example of an operator from this class. The operator $A$ given by the relations

$$
A u=-\Delta u+\rho^{\nu}(x) u, \quad \nu>2, \quad D(A)=C_{0}^{\infty}(\Omega)
$$

is essentially selfadjoint in $L^{2}(\Omega)$, i.e., its closure $\bar{A}$ is a selfadjoint operator in $L^{2}(\Omega), D(\bar{A})=W_{2}^{2}\left(\Omega, 1, \rho^{2 \nu}\right)([8], \S 6.4 .3)$ and $A$ has a purely pointwise spectrum. Moreover, $A$ is positive definite. A sequence of eigenfunctions $\left\{\varphi_{j}\right\}$ of the operator $A$ belongs to the space $S_{\rho(x)}(\Omega)$. It is also proved that $D\left(\bar{A}^{j}\right)=$ $W_{2}^{j}\left(\Omega, 1, \rho^{2 \nu j}\right)([8], \S 6.4 .3)$ and the space $S_{\rho(x)}(\Omega)$ is isomorphic to the space $D\left(\bar{A}^{\infty}\right)$, where the topology of the space $D\left(\bar{A}^{\infty}\right)$ is given by the sequence of Hilbertian norms $(7)$, while the topology of the space $S_{\rho(x)}(\Omega)$ by the sequence $\left\{\|\cdot\|_{n, \alpha}\right\}$. Therefore if we consider the equation

$$
-\Delta u+\rho^{\nu}(x) u=f
$$

in the Fréchet space $S_{\rho(x)}(\Omega)$ with the sequence of norms (19), then by virtue of proposition b) of Theorem 3 (see also [8], $\S 6.4 .3$ ) it has a unique solution for each $f \in S_{\rho(x)}(\Omega)$. If the sequence of eigenfunctions $\left\{\varphi_{j}\right\}$ is orthogonal in the space $L^{2}(\Omega)$, then the sequence of approximate solutions $\left\{u_{m}\right\}$ constructed by the extended Ritz method and given by equality (15) converges in the space $S_{\rho(x)}(\Omega)$ to a solution of equation $(21)$. 
Let us now give a concrete definition of the result in the one-dimensional case for the Hermite operator, i.e., for the harmonic oscillator

$$
A u=-u^{\prime \prime}(t)+t^{2} u
$$

without imposing additional boundary conditions. It is a selfadjoint and positive definite operator in the Hilbert space $L^{2}(R)$. According to [15], the Schwartz space $S(R)$ serves as the space $D\left(A^{\infty}\right)$ for the operator $A$. The eigenfunctions of the operator are Hermite functions (wave functions of the harmonic oscillator) ([16], p. 115), since

$$
\left(-\frac{d^{2}}{d t^{2}}+t^{2}\right) \varphi_{j}=(2 j+1) \varphi_{j}
$$

where $\varphi_{0}=\pi^{-1 / 4} e^{\frac{t^{2}}{2}}$ and

$$
\varphi_{j}(t)=\left(2^{j} j !\right)^{-1 / 2}(-1)^{j} \pi^{-1 / 4} e^{\frac{t^{2}}{2}}\left(\frac{d}{d t}\right)^{(j)} e^{-t^{2}} \quad(j \geq 1) .
$$

This means that $\lambda_{j}=2 j+1, j=1,2, \ldots$ The sequence $\left\{\varphi_{j}\right\}$ is the orthonormal basis in the space $L^{2}(R)$ and, by virtue of the nuclearity of the space $S(R)$, it is also the absolute basis in the latter space. Let us consider the space $S(R)$ with the sequence of Hilbertian norms

$$
\|h\|_{n}=\left(\|h\|^{2}+\left\|\left(-\frac{d^{2}}{d t^{2}}+t^{2}\right) h\right\|^{2}+\cdots+\left\|\left(-\frac{d^{2}}{d t^{2}}+t^{2}\right)^{n-1} h\right\|^{2}\right)^{1 / 2},
$$

where $\|\cdot\|$ is the norm of the space $L^{2}(R)$.

Let the operator $A^{\infty}$ be the restriction of the operator $A$ to the space $S(R) \subset$ $D(A)$ with the topology of the latter space taken into account. By virtue of (16), an approximate solution of the equation $A^{\infty} u=f$ has the form

$$
u_{m}=\sum_{j=1}^{m-1} \frac{\left(f, \varphi_{j}\right)}{2 j+1} \varphi_{j}
$$

where $\left\{\varphi_{j}\right\}$ are defined by equality (22). The sequence of approximate solutions $\left\{u_{m}\right\}$ converges to a solution of the equation $A^{\infty} u=f$ in the topology of the space $S(R)$.

The program realizing the convergence of a sequence of approximate solutions for various functions from the space $S(R)$ has been written in the PASCAL algorithmic language in cooperation with $\mathrm{S}$. Razmadze. The obtained numerical results have confirmed the above theoretical conclusions. Numerical analogues of approximate solutions (24) converge to a solution of the equation $A^{\infty} u=f$ for sufficiently large numbers of the norms from (23) for various functions from the space $S(R)$.

The obtained results can be applied to essentially selfadjoint and positive definite Legendre operators $A_{m, k}(2 k \leq m)([8], \S 7.4 .1)$ and Tricomi operators $B_{n, k}([8], \S 7.6 .3)$. These works also give the representations of spaces $D\left(\bar{A}_{m, k}^{\infty}\right)$ $([8], \S 7.4 .4)$ and $D\left(\bar{B}_{m, k}^{\infty}\right)([8], \S 7.6 .3)$. 
Let $H$ be a Hilbert space. Let us consider equation (3), where $K$ is the oneto-one compact selfadjoint operator. Let $K^{-1}$ be the selfadjoint operator having eigenvectors $\left\{\varphi_{j}\right\}$, generating an orthonormalized basis of the space $H$ and corresponding to the eigenvalues $\left\{\lambda_{j}\right\}\left(\left|\lambda_{1}\right| \leq\left|\lambda_{2}\right| \leq \ldots\right)$. This means that in the Hilbert space $H$, Hadamard's second condition of solution uniqueness is fulfilled, but the third condition of solution stability is not. By virtue of proposition $b$ ) of Theorem 3 the restriction $K^{-1}$ on the countable-Hilbert space $D\left(K^{-\infty}\right)$ is a continuous selfadjoint operator on the Fréchet space $D\left(K^{-\infty}\right)$. Moreover, by virtue of the remark made after the proof of Theorem 1.5, it follows from [16] that the space $D\left(K^{-\infty}\right)$ is everywhere dense in its strongly adjoint space $D\left(K^{-\infty}\right)_{\beta}^{\prime}$ which in this case is represented as the inductive limit of the increasing sequence of Hilbert spaces $\left\{\left(D\left(K^{-\infty}\right),\|\cdot\|_{n}\right)^{\prime}\right\}$. Therefore the operator $K^{-1}$ can be continuously extended to the spaces $D\left(K^{-\infty}\right)_{\beta}^{\prime}$. The extended operator $K^{-1}$ maps the space $D\left(K^{-\infty}\right)_{\beta}^{\prime}$ into and is continuous. Therefore we obtain the strong well-posedness of problem (3) as different from its weak well-posedness in [10], [11]. We have thereby proved that our next statement is true.

Theorem 6. Let $K: H \rightarrow H$ be the one-to-one compact and selfadjoint operator whose inverse operator $K^{-1}$ is selfadjoint and has a discrete spectrum. Then for each $f \in D\left(K^{-\infty}\right)_{\beta}^{\prime}$ there exists a unique and strongly stable solution of equation (3) in the space $D\left(K^{-\infty}\right)_{\beta}^{\prime}$.

Note that the operator $K^{-1}$ is continuous also when $D\left(K^{-\infty}\right)^{\prime}$ is equipped with a weak topology, which, by [11], means that problem (3) is weakly wellposed.

Let us now consider the equation

$$
R u=f,
$$

where $R: H \rightarrow M$ is the operator acting from the Hilbert space $H$ into the Hilbert space $M$. Let $u_{0}=R^{+} f$ be a generalized solution in the sense of Moore-Penrose ([22], p. 96), i.e., be an element of a minimal norm giving a minimal value of $\|R u-f\|$. In the literature we also encounter other names: a quasisolution in the sense of V. K. Ivanov [10] or a best approximate solution in the sense of [23]. An element $u_{0}=R^{+} f$ is a generalized solution if and only if $u_{0}$ satisfies the equation $R^{*} R u=R^{*} f$ and belongs to the subspace $\overline{\operatorname{Im} R^{*}}$.

Let now the operator $R: H \rightarrow M$ admit a singular decomposition, i.e., be represented in the form

$$
R u=\sum_{k=1}^{\infty} \sigma_{k}\left(u, f_{k}\right) g_{k},
$$

where $\sigma_{k}>0,\left\{f_{k}\right\}$ and $\left\{g_{k}\right\}$ are orthonormalized sequences in the spaces $H$ and $M$, respectively. Then, by virtue of $[22$, p. 99] we obtain the representation

$$
R^{+} f=\sum_{k=1}^{\infty} \sigma_{k}^{-1}\left(f, g_{k}\right) f_{k}, \quad f \in \operatorname{Im} R+(\operatorname{Im} R)^{\perp}
$$


The operator $R^{*} R u: H \rightarrow H$ has the form

$$
R^{*} R u=\sum_{k=1}^{\infty} \sigma_{k}^{2}\left(u, f_{k}\right) f_{k}
$$

and is a selfadjoint and only positive operator. If the sequence $\left\{\sigma_{k}\right\}$ is bounded (resp. $\sigma_{k} \searrow 0$, resp. $\sigma_{k} \nearrow \infty$ ), then the operator $R^{*} R$ is a continuous (resp. compact, resp. positive definite with a purely pointwise spectrum) selfadjoint operator.

In the last case, let us consider the Fréchet space $D\left(\left(R^{*} R\right)^{\infty}\right.$ with the sequence of norms

$$
\|x\|_{n}=\left(\|x\|^{2}+\left\|R^{*} R x\right\|^{2}+\cdots+\left\|\left(R^{*} R\right)^{n-1} x\right\|^{2}\right)^{1 / 2}
$$

generated by the scalar products

$$
(x, y)_{n}=(x, y)+\left(R^{*} R x, R^{*} R y\right)+\cdots+\left(\left(R^{*} R\right)^{n-1} x,\left(R^{*} R\right)^{n-1} y\right) .
$$

By virtue of the above reasoning, the restriction of the operator $R^{*} R$ to the Fréchet space $D\left(\left(R^{*} R\right)^{\infty}\right)$ is denoted by $\left(R^{*} R\right)^{\infty}$ and is a selfadjoint and positive definite operator on the Fréchet space $D\left(\left(R^{*} R\right)^{\infty}\right)$. We use $(15)$ to solve the equation $\left(R^{*} R\right)^{\infty} u=R^{*} f$ in the Fréchet space $D\left(\left(R^{*} R\right)^{\infty}\right)$ and obtain that if $R^{*} f \in D\left(\left(R^{*} R\right)^{\infty}\right)$, the sequence of approximate solutions

$$
u_{m}=\sum_{k=1}^{m} \sigma_{k}^{-2}\left(R^{*} f, f_{k}\right) f_{k}, \text { where } R^{*} f=\sum_{k=1}^{\infty} \sigma_{k}\left(f, g_{k}\right) f_{k},
$$

converges to $R^{+} f$ in the Fréchet space $D\left(\left(R^{*} R\right)^{\infty}\right)$.

\section{ACKNOWLEDGEMENT}

The research was supported by Grant 1.20.04 of the Georgian Academy of Sciences.

\section{REFERENCES}

1. E. KRAmar, Locally convex topological vector spaces with Hilbertian seminorms. Rev. Roumaine Math. Pures Appl. 26(1981), No. 1, 55-62.

2. E. Kramar, Linear operators in H-locally convex spaces. Rev. Roumaine Math. Pures Appl. 26(1981), No. 1, 63-77.

3. D. N. Zarnadze and S. A. Tsotniashvili, On the existence of a solution of an equation with a symmetric and positive definite operator in some Fréchet spaces. Rep. Enlarged Sess. Semin. I. Vekua Inst. Appl. Math. 10(1995), No. 1, 87-90.

4. D. N. Zarnadze and S. A. Tsotniashvili, On the extension of Ritz's method for an equation with a symmetric and positive definite operator in some Fréchet spaces. Rep. Enlarged Sess. Semin. I. Vekua Inst. Appl. Math. 10(1995), No. 3, 81-83.

5. S. Tsotniashvili, On the extension of selfadjoint operators from Hilbert spaces to Fréchet-Hilbert spaces. Bull. Georgian Acad. Sci. 153(1996), No. 3, 338-341.

6. Ju. M. BereZanskiI , Selfadjoint operators in spaces of functions of an infinite number of variables. (Russian) Naukova Dumka, Kiev, 1978.

7. H. TRIEBEL, Nukleare Funktionenräume und singuläre elliptische Differentialoperatoren. Studia Math. 38(1970), 285-311. 
8. H. Triebel, Interpolation theory, function spaces, differential operators. North-Holland Mathematical Library, 18. North-Holland Publishing Co., Amsterdam-New York, 1978.

9. M. REed and B. Simon, Methods of modern mathematical physics. II. Fourier analysis, self-adjointness. Academic Press [Harcourt Brace Jovanovich, Publishers], New YorkLondon, 1975.

10. V. K. Ivanov, Hadamard conditions for well-posedness in spaces of generalized functions. (Russian) Sibirsk. Mat. Zh. 28(1987), No. 6, 53-59.

11. V. K. Ivanov and I. V. MeLnikova, New generalized functions and weak well-posedness of operator problems. Complex analysis and generalized functions (September, 1991), 141147, Varna, 1991.

12. D. ZARNADZE, On spline algorithms and best approximations in finite codimensional subspaces. Rep. Enlarged Sess. Semin. I. Vekua Inst. Appl. Math. 14(1999), No. 3, 91-94.

13. D. N. Zarnadze and S. A. Tsotniashvili, On generalized interpolating splines in the spaces of differentiable local integrable functions. J. Intelekti 2(16)(2003), 11-15.

14. K. Moren, Methods of Hilbert space. (Russian) (Translated from the Polish) Mir, Moscow, 1965.

15. A. Pietsch, Über die Erzeugung von $(F)$-Räumen durch selbstadjungierte Operatoren. Math. Ann. 164(1966), 219-224.

16. V. S. Vladimirov, Generalized functions in mathematical physics. (Russian) Current Problems in Physics and Technology Series, Nauka, Moscow, 1976.

17. D. N. Zarnadze, Some topological and geometric properties of Fréchet-Hilbert spaces. (Russian) Izv. Ross. Akad. Nauk Ser. Mat. 56(1992), No. 5, 1001-1020; English transl.: Russian Acad. Sci. Izv. Math. 41(1993), No. 2, 273-288.

18. S. Prössdorf, Einige Klassen singulärer Gleichungen. Mathematische Reihe, Band 46. Birkhäuser Verlag, Basel-Stuttgart, 1974; Russian transl.: Mir, Moscow, 1979.

19. D. N. ZARnADZE, On a generalization of the least squares method for operator equations in some Frchet spaces. (Russian) Izv. Ross. Akad. Nauk Ser. Mat. 59(1995), No. 5, 59-72; English transl.: Izv. Math. 59(1995), No. 5, 935-948.

20. J. F. Traub, G. W. Wasilkowski, and H. Woźniakowski, Information-based complexity. Computer Science and Scientific Computing. Academic Press, Inc., Boston, MA, 1988.

21. D. N. ZARnAdZe, Fréchet spaces with some classes of proximinal subspaces. (Russian) Izv. Akad. Nauk SSSR Ser. Mat. 50(1986), No. 4, 711-725; English transl.: Math. USSR, Izv. 29(1987), 67-79.

22. F. NAtTerer, The mathematics of computerized tomography. Classics in Applied Mathematics, 32. Society for Industrial and Applied Mathematics (SIAM), Philadelphia, PA, 2001.

23. W. J. Kammerer and M. Z. NAshed, Iterative methods for best approximate solutions of linear integral equations of the first and second kinds. J. Math. Anal. Appl. 40(1972), $547-573$.

(Received 20.05.2005)

Authors' addresses:

S. Tsotniashvili

Tskhinvali State University

2, Besiki St., Gori 1400

Georgia

E-mail: eredvi@yandex.ru
D. Zarnadze

N. Muskhelishvili Inst. Comput. Math. Georgian Academy of Sciences

8, Akuri St, Tbilisi 0193, Georgia

E-mail: z-dato@rambler.ru 